\title{
Damped, direction-dependent multigrid for hypersonic flow computations *
}

\author{
B. Koren and P.W. Hemker \\ Centre for Mathematics and Computer Science, P.O. Box 4079, 1009 AB Amsterdam, Netherlands
}

\begin{abstract}
Koren, B. and P.W. Hemker, Damped, direction-dependent multigrid for hypersonic flow computations, Applied Numerical Mathematics 7 (1991) 309-328.

A nonlinear multigrid technique with improved robustness is developed for the solution of the steady Euler equations. The system of nonlinear equations is discretized by an upwind finite volume method. Collective symmetric point Gauss-Seidel relaxation is applied as the standard smoothing technique. In case of failure of the point relaxation, a switch is made to a local evolution technique. The novel robustness improvements to the nonlinear multigrid method are a local damping of the restricted defect, a global upwind prolongation of the correction and a global upwind restriction of the defect. The defect damping operator is derived from a two-grid convergence analysis. The upwind prolongation operator is made such that it is consistent with the upwind finite volume discretization. It makes efficient use of the P-variant of Osher's approximate Riemann solver. The upwind restriction operator is an approximate adjoint of the upwind prolongation operator. Satisfactory convergence results are shown for the computation of a hypersonic launch and reentry flow around a blunt forebody with canopy. For the test cases considered, it appears that the improved multigrid method performs significantly better than a standard nonlinear multigrid method. For all test cases considered it appears that the most significant improvement comes from the upwind prolongation, rather than from the upwind restriction and the defect damping.
\end{abstract}

Keywords. Nonlinear multigrid method, steady Euler equations, hypersonic flows.

\section{Introduction}

\subsection{Governing equations}

The flow equations considered are the steady, 2D Euler equations

$$
\frac{\partial}{\partial x}\left[\begin{array}{c}
\rho u \\
\rho u^{2}+p \\
\rho u \nu \\
\rho u(e+p / \rho)
\end{array}\right]+\frac{\partial}{\partial y}\left[\begin{array}{c}
\rho \nu \\
\rho \nu u \\
\rho \nu^{2}+p \\
\rho \nu(e+p / \rho)
\end{array}\right]=0,
$$

with the total energy satisfying, assuming a perfect gas,

$$
e=\frac{1}{\gamma-1} \frac{p}{\rho}+\frac{1}{2}\left(u^{2}+\nu^{2}\right)
$$

\footnotetext{
* This work was supported by the European Space Agency (ESA), through Avions Marcel Dassault-Bréguet Aviation (AMD-BA).
} 
So far, real gas effects are not taken into account. The specific heat ratio $\gamma$ of the di-atomic gas considered is assumed to be constant and determined by fully excited translational and rotational energies only. (Though it could easily be replaced by a function ranging from zero up to the full equipartition value, the vibrational energy is assumed to be zero.)

\subsection{Existing computational method}

For a description of the existing computational method which is taken as a point of departure, we refer to $[3,4]$. Here we give an overview of its main characteristics only.

\section{Discretization method}

Discretized equations are obtained by dividing the computational domain $\Omega$ into quadrilateral finite volumes $\Omega_{i, j}$ and by requiring that the conservation laws, (1.1a) in integral form, hold for each finite volume separately. This discretization requires an evaluation of the convective flux vector at each cell face. For this, we prefer an upwind approach which follows the Godunov principle [2]. The 1D Riemann problem thus arising at each cell face is solved in an approximate way by using Osher's scheme [5] in its P-variant [3]. The left and right state in the 1D Riemann problem (which determine the accuracy of the convective discretization) are simply taken equal to those in the corresponding adjacent volumes, leading to first-order accuracy.

\section{Solution method}

For the solution of the nonlinear system of first-order accurate discretized Euler equations, we apply collective symmetric point Gauss-Seidel relaxation, accelerated by nonlinear multigrid. The solution process is started by nested iteration. In the relaxation method one or more exact Newton steps are used for the collective update of the four state vector components in each finite volume. The method summarized so far is that presented in [3]. The possible extension, as presented in [4], is the following. If necessary, the success (or failure) of the relaxation method may be continuously checked by monitoring the error behaviour and the physical relevance of the iterates. In case of failure of the relaxation method, a switch is made to a fully explicit evolution technique to solve the system of nonlinear equations in the particular cell where problems arise. This solution method with enhanced robustness still does not require any tuning of parameters. We proceed by giving a summary of our nonlinear multigrid and nested iteration method.

Nested grids are applied such that each finite volume on a coarse grid is the union of $2 \times 2$ volumes on the next finer grid. Let $\Omega_{1}, \ldots, \Omega_{l-1}, \Omega_{l}, \Omega_{l+1}, \ldots, \Omega_{L}$ be a sequence of such nested grids, with $\Omega_{1}$ the coarsest and $\Omega_{L}$ the finest grid, and let $N_{l}\left(q_{l}\right)=r_{l}$ denote the nonlinear system of first-order discretized Euler equations on $\Omega_{l}$, with $r_{l}$ a possibly nonzero right-hand side related to the multigrid iteration. Then a single nonlinear multigrid cycle and the nested iteration, as applied in the existing solution method, are defined in the following way.

\section{Nonlinear multigrid iteration}

- Apply on $\Omega_{l} n_{\text {pre }}$ pre-relaxation sweeps to $N_{l}\left(q_{l}\right)=r_{l}$.

- Compute the defect $d_{l}=N_{l}\left(q_{l}\right)-r_{l}$ and restrict it to $\Omega_{l-1}: d_{l-1}=I_{l}^{l-1} d_{l}$, where $I_{l}^{l-1}$ is a restriction operator for right-hand sides. 
- Compute on the next coarser grid $\Omega_{l-1}$ the right-hand side $r_{l-1}=N_{l-1}\left(q_{l-1}\right)-d_{l-1}$. For the initial estimate of $q_{l-1}$, we use the latest obtained $q_{l-1}$.

- Approximate the solution of $N_{l-1}\left(q_{l-1}\right)=r_{l-1}$ by the application of $n_{\sigma}$ nonlinear multigrid cycles. Denote the approximation obtained as $\tilde{q}_{l-1}$.

- Correct the current solution by $q_{l}=q_{l}+\tilde{I}_{l-1}^{l}\left(\tilde{q}_{l-1}-q_{l-1}\right)$, where $\tilde{I}_{l-1}^{l}$ is a linear prolongation operator for solutions.

- Apply on $\Omega_{l} n_{\text {post }}$ post-relaxation sweeps to $N_{l}\left(q_{l}\right)=r_{l}$.

For $l=1$, the coarse grid correction is skipped of course. For the restriction operator $I_{l}^{l-1}$ and the prolongation operator $\tilde{I}_{l-1}^{l}$ we take

$$
\begin{aligned}
\left(r_{l-1}\right)_{i, j}=\left(I_{l}^{l-1} r_{l}\right)_{i, j} & \\
=\left(r_{l}\right)_{2 i-1,2 j-1}+\left(r_{l}\right)_{2 i-1,2 j}+\left(r_{l}\right)_{2 i, 2 j-1}+\left(r_{l}\right)_{2 i, 2 j} & \\
\left(\tilde{I}_{l-1}^{l} q_{l-1}\right)_{2 i-1,2 j-1}=\left(\tilde{I}_{l-1}^{l} q_{l-1}\right)_{2 i-1,2 j}=\left(\tilde{I}_{l-1}^{l} q_{l-1}\right)_{2 i, 2 j-1} & =\left(\tilde{I}_{l-1}^{l} q_{l-1}\right)_{2 i, 2 j} \\
& =\left(q_{l-1}\right)_{i, j} .
\end{aligned}
$$

If not mentioned otherwise, for $n_{\sigma}$ and $n_{\text {pre }}, n_{\text {post }}$ we use at each level $l: n_{\sigma}=1$ and $n_{\text {pre }}=n_{\text {post }}=1$; i.e. as nonlinear multigrid cycles we use V-cycles with a single pre- and post-relaxation sweep per level.

\section{Nested iteration}

- Choose a (possibly crude) initial estimate $q_{1}$.

- Improve $q_{1}$ by a single nonlinear multigrid cycle as just defined above.

- Prolongate the improved approximation $q_{1}$ to $\Omega_{2}$, yielding an initial estimate for $q_{2}$.

- Improve $q_{2}$ by a single nonlinear multigrid cycle as defined above.
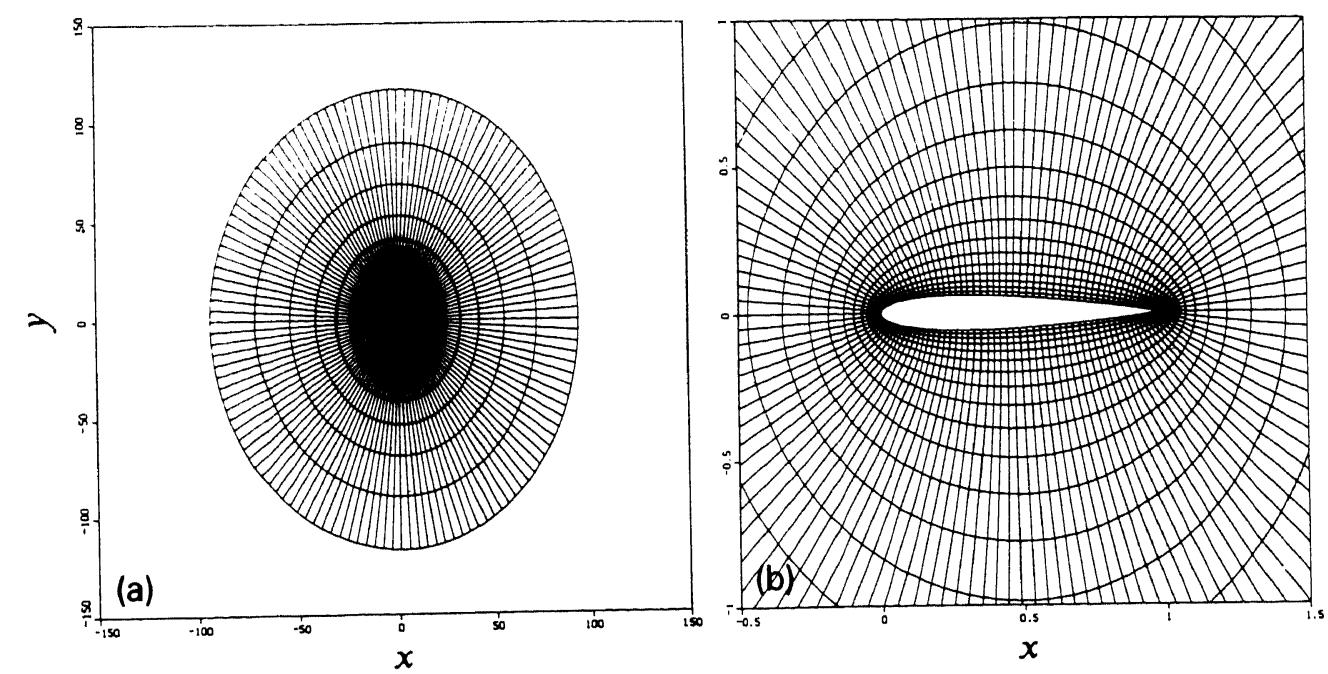

Fig. 1. (128 $\times 32)$-grid $\left(\Omega_{5}\right)$ NACA0012-airfoil: (a) in full, (b) in detail. 
Continue the previous process until an initial estimate for $q_{L}$ has been obtained by prolongation of $q_{L-1}$.

The prolongation operator for obtaining the first approximation on each next finer grid may be the piecewise constant operator $(1.2 b)$ or-preferably-a more accurate operator (for instance a bilinear operator).

\subsection{Results}

To give a quick impression of the performance of our existing multigrid method, we consider: (i) the NACA0012-airfoil at $M_{\infty}=0.63, \alpha=2^{\circ}$ (smooth subsonic flow) and at $M_{\infty}=0.85$, $\alpha=1^{\circ}$ (non-smooth transonic flow), and (ii) a blunt forebody with canopy at $M_{\infty}=8.15, \alpha=0^{\circ}$ (non-smooth hypersonic flow). For details about the geometry of the latter body, we refer to [4]. As finest finest-grid for the NACA0012-airfoil we consider the $128 \times 32$ O-type grid given in Fig. 1. For the blunt body, as finest finest-grid so far, we consider the $64 \times 32$ C-type grid given in Fig. 2.

\subsubsection{Smooth subsonic and non-smooth transonic flow}

For the NACA0012-flows we obtain the multigrid performance given in Fig. 3. In both graphs, the residual ratio along the vertical axis is the ratio

$$
\sum_{k=1}^{4}\left|\left(N_{L}\left(q_{L}^{n}\right)\right)_{k}\right| / \sum_{k=1}^{4}\left|\left(N_{L}\left(q_{L}^{1}\right)\right)_{k}\right|, \quad L=3,4,5
$$

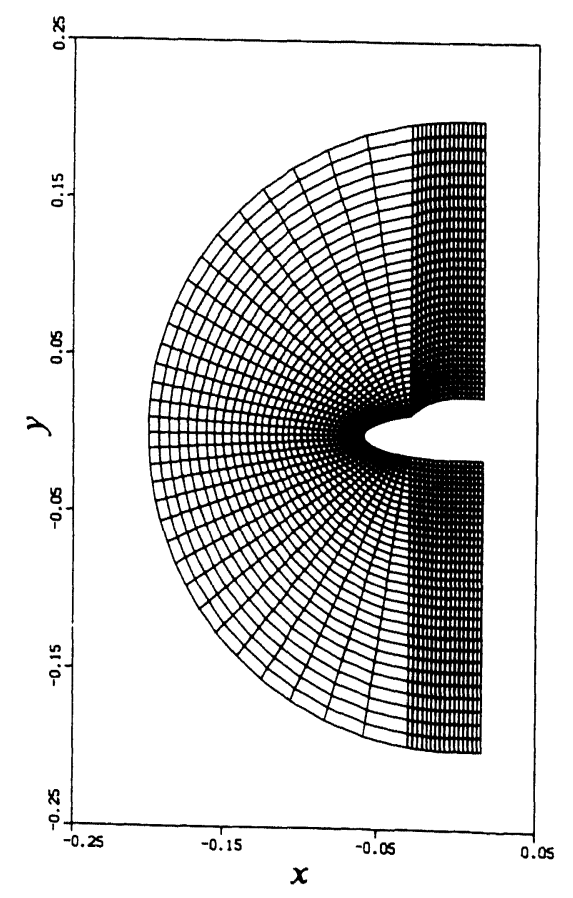

Fig. 2. $(64 \times 32)$-grid $\left(\Omega_{5}\right)$ blunt forebody with canopy. 

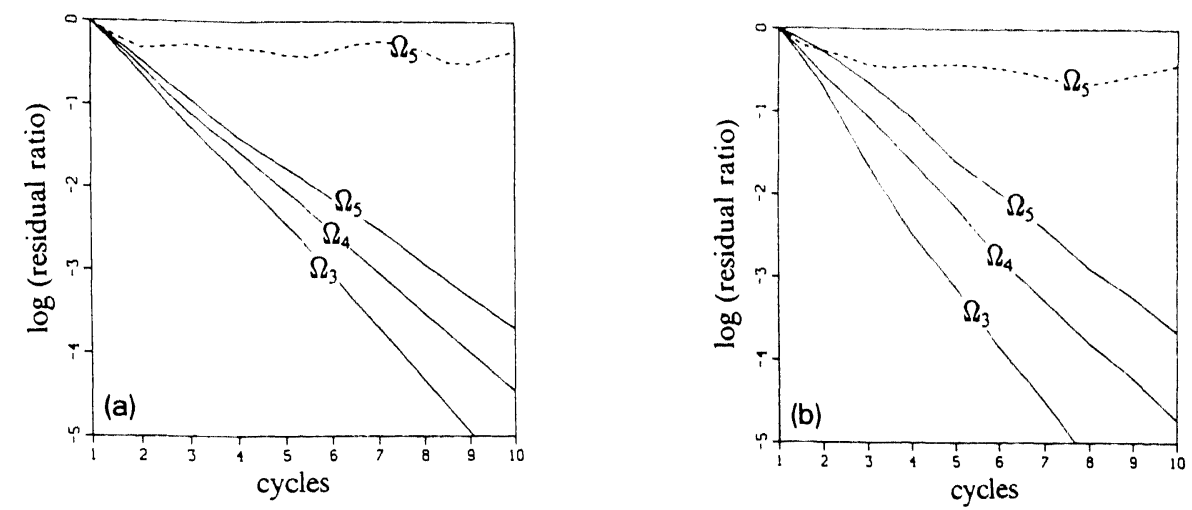

Fig. 3. Multigrid convergence behaviour, NACA0012-airfoil (a) at $M_{\infty}=0.63, \alpha=2^{\circ}$, (b) at $M_{\infty}=0.85, \alpha=1^{\circ}$.

(Dashed lines: single-grid; solid lines: multigrid.)

versus the number of cycles performed, one multigrid cycle being a V-cycle with $n_{\text {pre }}=n_{\text {post }}=1$, $\forall l$, and, for $\Omega_{5}$ only, one single-grid cycle being the equivalent number of finest-grid relaxation sweeps. $\left|\left(N_{L}\left(q_{L}^{n}\right)\right)_{k}\right|$ denotes the summation-over all volumes at $\Omega_{L}$-of the absolute values of the $k$ th component in the first-order Euler defects, with $q_{L}^{n}$ denoting the solution at $\Omega_{L}$ after the $n$th multi- or single-grid cycle. Considering the single-grid convergence histories, for both the smooth subsonic case and the non-smooth transonic case, the effectiveness of the multigrid method is clear.

\subsubsection{Non-smooth hypersonic flow}

For both previous airfoil flows, the switched-relaxation-evolution technique does not need to be invoked. However, for the hypersonic blunt body flow, the latter technique must be used. (Without this technique, already in the first relaxation sweep on the coarsest grid, the solution process breaks down.) For a similar hypersonic flow, in [4] we have already presented single-grid convergence results obtained with the switched-relaxation-evolution approach. With this approach, for all cases considered in [4] we obtain convergence. Further, it appears-as was

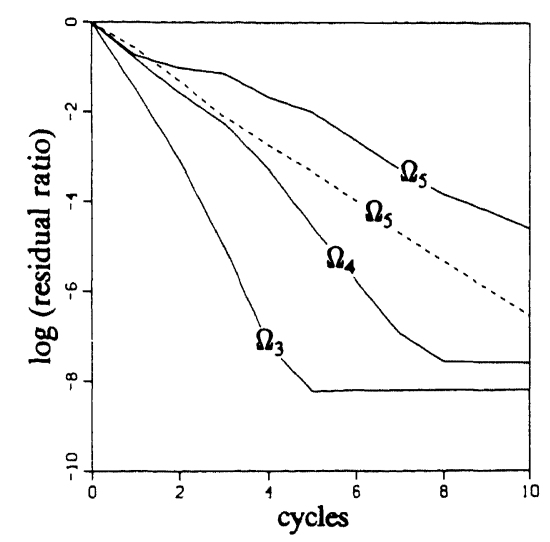

Fig. 4. Multigrid convergence behaviour, blunt forebody with canopy at $M_{\infty}=8.15, \alpha=0^{\circ}$. (Dashed line: single-grid; solid lines: multigrid.) 
expected - that the evolution technique makes itself superfluous in the course of the iteration process. For the present hypersonic test case, we have the same single-grid experience. Unfortunately, with the switched-relaxation-evolution technique combined with the existing multigrid method, no satisfactory results are obtained; see Fig. 4 , with $q_{L}^{0}$ being the approximate solution obtained by the nested iteration. Standard changes to the multigrid algorithm, such as for instance the replacement of V-cycles $\left(n_{\sigma}=1\right)$ by W-cycles $\left(n_{\sigma}=2\right)$ do not help. In the next section we present novel changes to the multigrid algorithm which will improve the poor results presented in Fig. 4.

\section{Improved multigrid method}

Applying multigrid in the standard way as described in Section 1.2, we experience that for hypersonic test cases, local coarse-to-fine grid corrections may be transferred which sweep the corresponding fine grid iterates out of the attraction domain of the pure relaxation technique and even out of that of the switched-relaxation-evolution technique. The cause of these problems may be either the coarse grid corrections themselves, or the prolongation operator, or the combination of both. Therefore, in the present paper, to avoid possibly bad coarse grid corrections, we present a local damping technique for the restricted defects and hence-implicitly-a local damping technique for the coarse grid corrections. To avoid a possibly bad correction transfer, we present an alternative prolongation: a direction-dependent prolongation.

\subsection{Defect damping}

In [1], De Zeeuw reports a serious lack of robustness of standard nonlinear multigrid applied to a test case described by the steady, 1D semiconductor equations. De Zeeuw meets the quest for greater robustness by a local damping of the restricted defect. Though the way in which this damping is introduced in [1] is not yet very convincing from a theoretical point of view (only very little evidence is given for the amount of damping to be applied), it certainly is convincing from an experimental point of view. It leads to a significantly more robust multigrid method. For a class of elliptic problems, a likewise successful, but basically different damping technique for improving the robustness of nonlinear multigrid, is that proposed by Reusken [6]. Instead of locally damping the restricted defect, Reusken proposes to damp globally (i.e. uniformly) the coarse grid correction, the amount of damping to be applied being prescribed by fairly rigorous theory. For the present hypersonic flow computations, we prefer the type of damping as proposed by De Zeeuw, (i) because it is not restricted to a specific class of elliptic problems (not even to elliptic problems in general), (ii) because of its a-priori character (a-posteriori damping like that in [6] may already be too late), and (iii) because of its local application (global damping like that in [6] may strongly reduce the positive effects of the coarse grid correction).

The local defect damping to be applied is now introduced by deriving the two-grid amplification operator. Let

$$
N_{l}\left(q_{l}\right)=r_{l}
$$


denote a nonlinear system of fine grid equations that we want to solve. Then the corresponding $(n+1)$ st coarse grid problem $(n=0,1, \ldots, N)$ to be solved, reads

$$
N_{l-1}\left(q_{l-1}^{n+1}\right)=N_{l-1}\left(q_{l-1}^{n}\right)-S_{l-1} I_{l}^{l-1}\left(N_{l}\left(q_{l}^{n+1 / 3}\right)-r_{l}\right), \quad n=0,1, \ldots, N,
$$

with $S_{l-1}$ denoting the operator for the defect damping, with $q_{l}^{n+1 / 3}$ denoting the fine grid iterate as obtained after the (fine grid) pre-relaxation, and with $q_{l-1}^{n}$ and $q_{l-1}^{n+1}$ denoting the coarse grid iterates before the (coarse grid) pre-relaxation and after the (coarse grid) post-relaxation, respectively. With (2.1) and by linearization (neglecting higher-order terms), from (2.2) we derive the relation

$$
\begin{aligned}
& q_{l-1}^{n+1}-q_{l-1}^{n}=-\left[\frac{\mathrm{d} N_{l-1}\left(q_{l-1}^{n}\right)}{\mathrm{d} q_{l-1}}\right]^{-1} S_{l-1} I_{l}^{l-1} \frac{\mathrm{d} N_{l}\left(q_{l}^{n+1 / 3}\right)}{\mathrm{d} q_{l}}\left(q_{l}^{n+1 / 3}-q_{l}^{*}\right), \\
& n=0,1, \ldots, N,
\end{aligned}
$$

in which $q_{l}^{*}$ denotes the fully converged solution of (2.1). Considering for the coarse grid correction $q_{l-1}^{n+1}-q_{l-1}^{n}$ also the relation

$$
\tilde{I}_{l-1}^{l}\left(q_{l-1}^{n+1}-q_{l-1}^{n}\right)=\left(q_{l}^{n+2 / 3}-q_{l}^{n+1 / 3}\right), \quad n=0,1, \ldots, N,
$$

with $q_{l}^{n+2 / 3}$ denoting the fine grid iterate before the (fine grid) post-relaxation, it follows the two-grid convergence result

$$
\begin{aligned}
& q_{l}^{n+2 / 3}-q_{l}^{*}=\left[I_{l}-\tilde{I}_{l-1}^{l}\left[\frac{\mathrm{d} N_{l-1}\left(q_{l-1}^{n}\right)}{\mathrm{d} q_{l-1}}\right]^{-1} S_{l-1} I_{l}^{l-1} \frac{\mathrm{d} N_{l}\left(q_{l}^{n+1 / 3}\right)}{\mathrm{d} q_{l}}\right]\left(q_{l}^{n+1 / 3}-q_{l}^{*}\right), \\
& \quad n=0,1, \ldots, N,
\end{aligned}
$$

in which $I_{l}$ denotes the identity operator on $\Omega_{l}$. From (2.5), it is clear that in case the property

$$
\frac{\mathrm{d} N_{l-1}\left(q_{l-1}^{n}\right)}{\mathrm{d} q_{l-1}}=I_{l}^{l-1} \frac{\mathrm{d} N_{l}\left(q_{l}^{n+1 / 3}\right)}{\mathrm{d} q_{l}} \tilde{I}_{l-1}^{l}
$$

is satisfied, (2.5) implies

$$
\begin{aligned}
& I_{l}^{l-1} \frac{\mathrm{d} N_{l}\left(q_{l}^{n+1 / 3}\right)}{\mathrm{d} q_{l}}\left(q_{l}^{n+2 / 3}-q_{l}^{*}\right)=\left(I_{l-1}-S_{l-1}\right) I_{l}^{l-1} \frac{\mathrm{d} N_{l}\left(q_{l}^{n+1 / 3}\right)}{\mathrm{d} q_{l}}\left(q_{l}^{n+1 / 3}-q_{l}^{*}\right), \\
& \quad n=0,1, \ldots, N,
\end{aligned}
$$

indicating that for optimal two-grid convergence no damping should be applied $\left(S_{l-1}=I_{l-1}\right)$. However, in hypersonic flow computations, $q_{l-1}^{n}$ and $q_{l}^{n+1 / 3}$ may strongly differ from each other and as a consequence also both Jacobians in (2.6). For example, a hypersonic shock wave which is detached on $\Omega_{l}$, may easily be attached on $\Omega_{l-1}$, with as a probable consequence that there, locally (2.6) is not satisfied at all. If (2.6) is not satisfied, in particular if this is only very locally the case, damping of the restricted defect at those places might be very useful. For optimal 
two-grid convergence, from (2.5) we derive as local damping factor for the defect in finite volume $\left(\Omega_{l-1}\right)_{i, j}$ to be applied in the $(n+1)$ st multigrid cycle:

$$
\begin{aligned}
& \left(S_{l-1}^{n+1}\right)_{i, j}=\min \left[1, \frac{\left\|\left(N_{l-1}^{\prime}\right)_{i, j}\right\|}{\max \left[\left\|\left(N_{l}^{\prime}\right)_{2 i-1,2 j-1}\right\|,\left\|\left(N_{l}^{\prime}\right)_{2 i-1,2 j}\right\|,\left\|\left(N_{l}^{\prime}\right)_{2 i, 2 j-1}\right\|,\left\|\left(N_{l}^{\prime}\right)_{2 i, 2 j}\right\|\right]}\right], \\
& n=0,1, \ldots, N,
\end{aligned}
$$

with $N_{l-1}^{\prime} \equiv \mathrm{d} N_{l-1}\left(q_{l-1}^{n}\right) / \mathrm{d} q_{l-1}$, with $N_{l}^{\prime} \equiv \mathrm{d} N_{l}\left(q_{l}^{n+1 / 3}\right) / \mathrm{d} q_{l}$ and with $\|\cdot\|$ some matrix norm. Notice that the local damping factor (2.8) is more or less the $2 \mathrm{D}$ equivalent of the $1 \mathrm{D}$ damping introduced in [1]. To see if some additional gain can be obtained by also allowing local defect amplification, in a numerical experiment we will also consider

$$
\begin{aligned}
& \left(S_{l-1}^{n+1}\right)_{i, j}=\frac{\left\|\left(N_{l-1}^{\prime}\right)_{i, j}\right\|}{\max \left[\left\|\left(N_{l}^{\prime}\right)_{2 i-1,2 j-1}\right\|,\left\|\left(N_{l}^{\prime}\right)_{2 i-1,2 j}\right\|,\left\|\left(N_{l}^{\prime}\right)_{2 i, 2 j-1}\right\|,\left\|\left(N_{l}^{\prime}\right)_{2 i, 2 j}\right\|\right]}, \\
& \quad n=0,1, \ldots, N .
\end{aligned}
$$

At convergence of the solution, the defect multiplication, both in case of (2.8) and in case of (2.9), will also have converged. However, as opposed to e.g. the correction damping proposed in [6], the present defect multiplication will not have vanished at convergence, neither in case of (2.8), nor in case of (2.9).

\subsection{Direction-dependent grid transfer operators}

\subsubsection{Prolongation}

The standard, piecewise constant correction prolongation may be illustrated as in Fig. 5. In mathematical terms, solution correction by means of the piecewise constant correction prolonga-

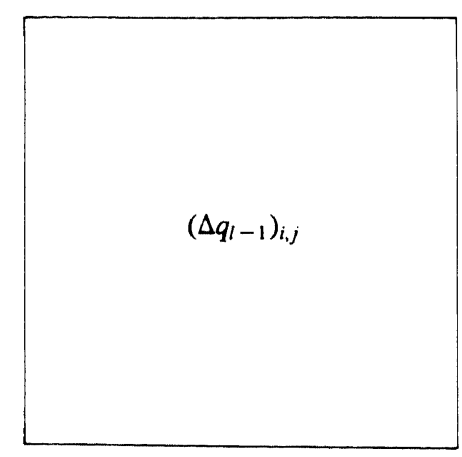

a. $\left(\Omega_{l-1}\right)_{i, j}$.

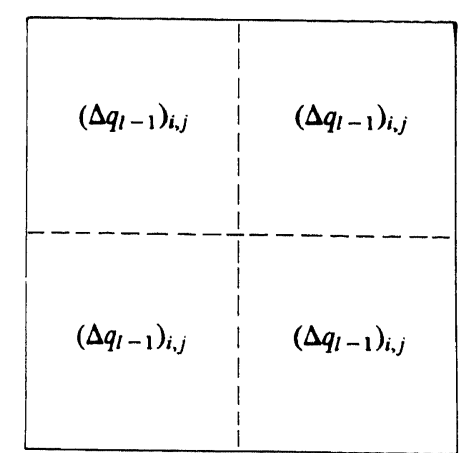

b. $\left(\Omega_{l}\right)_{2 i-1,2 j-1},\left(\Omega_{l}\right)_{2 i-1,2 j},\left(\Omega_{l}\right)_{2,2 j-1},\left(\Omega_{l}\right)_{2,2 j}$.

Fig. 5. Coarse grid finite volume with corresponding next-finer grid volumes and corresponding piecewise constant corrections. 


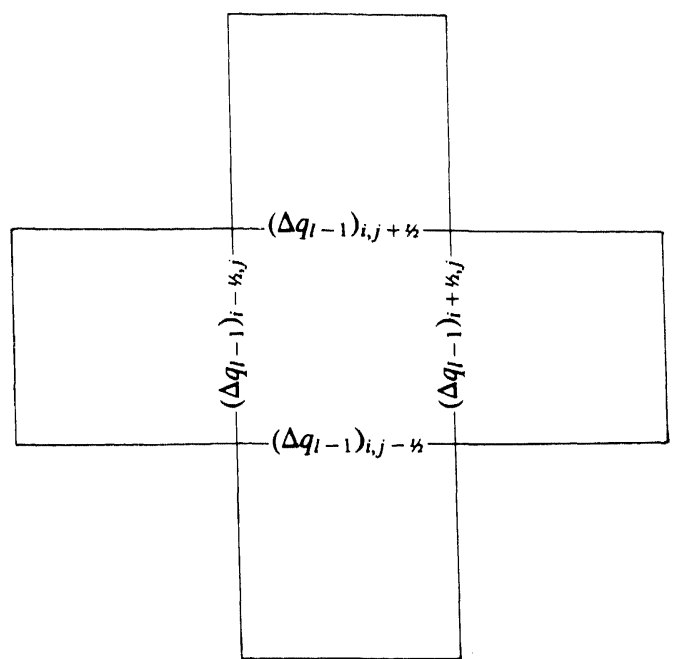

a. $\left(\Omega_{l-1}\right)_{i, j}$.

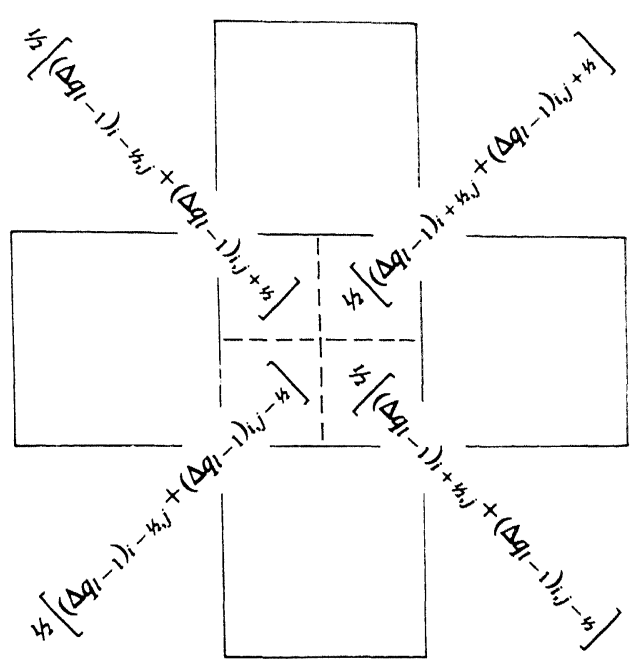

b. $\left(\mathbf{\Omega}_{l}\right)_{2 i-1,2 j-1},\left(\mathbf{\Omega}_{l}\right)_{2 i-1,2 j},\left(\mathbf{\Omega}_{l}\right)_{2,2 j-1},\left(\mathbf{\Omega}_{l}\right)_{2,2 j}$.

Fig. 6. Coarse grid finite volume with corresponding next-finer grid volumes and corresponding direction-dependent corrections.

tion may be written as

$$
\begin{aligned}
& \left(q_{l}^{\text {new }}\right)_{2 i-1,2 j-1}=\left(q_{l}^{\text {old }}\right)_{2 i-1,2 j-1}+\left(\Delta q_{l-1}\right)_{i, j}, \\
& \left(q_{l}^{\text {new }}\right)_{2 i-1,2 j}=\left(q_{l}^{\text {old }}\right)_{2 i-1,2 j}+\left(\Delta q_{l-1}\right)_{i, j}, \\
& \left(q_{l}^{\text {new }}\right)_{2 i, 2 j-1}=\left(q_{l}^{\text {old }}\right)_{2 i, 2 j-1}+\left(\Delta q_{l-1}\right)_{i, j}, \\
& \left(q_{l}^{\text {new }}\right)_{2 i, 2 j}=\left(q_{l}^{\text {old }}\right)_{2 i, 2 j}+\left(\Delta q_{l-1}\right)_{i, j},
\end{aligned}
$$

with

$$
\left(\Delta q_{l-1}\right)_{i, j}=\left(q_{l-1}^{\text {new }}\right)_{i, j}-\left(q_{l-1}^{\text {old }}\right)_{i, j}
$$

The direction-dependent correction prolongation that we propose now can be illustrated as in Fig. 6. In mathematical terms-analogous to (2.10)-solution correction by means of this direction-dependent prolongation is written as

$$
\begin{aligned}
& \left(q_{l}^{\text {new }}\right)_{2 i-1,2 j-1}=\left(q_{l}^{\text {old }}\right)_{2 i-1,2 j-1}+\frac{1}{2}\left[\left(\Delta q_{l-1}\right)_{i-1 / 2, j}+\left(\Delta q_{l-1}\right)_{i, j-1 / 2}\right], \\
& \left(q_{l}^{\text {new }}\right)_{2 i-1,2 j}=\left(q_{l}^{\text {old }}\right)_{2 i-1,2 j}+\frac{1}{2}\left[\left(\Delta q_{l-1}\right)_{i-1 / 2, j}+\left(\Delta q_{l-1}\right)_{i, j+1 / 2}\right], \\
& \left(q_{l}^{\text {new }}\right)_{2 i, 2 j-1}=\left(q_{l}^{\text {old }}\right)_{2 i, 2 j-1}+\frac{1}{2}\left[\left(\Delta q_{l-1}\right)_{i+1 / 2, j}+\left(\Delta q_{l-1}\right)_{i, j-1 / 2}\right], \\
& \left(q_{l}^{\text {new }}\right)_{2 i, 2 j}=\left(q_{l}^{\text {old }}\right)_{2 i, 2 j}+\frac{1}{2}\left[\left(\Delta q_{l-1}\right)_{i+1 / 2, j}+\left(\Delta q_{l-1}\right)_{i, j+1 / 2}\right],
\end{aligned}
$$


with the four fine grid cell centre corrections (Fig. 6(b)) defined as central averages of the coarse grid cell face corrections (Fig. 6(a)). The coarse grid cell face corrections are defined by

$$
\begin{aligned}
& \left(\Delta q_{l-1}\right)_{i-1 / 2, j}=\left(q_{l-1}^{\text {new }}\right)_{i-1 / 2, j}-\left(q_{l-1}^{\text {old }}\right)_{i-1 / 2, j}, \\
& \left(\Delta q_{l-1}\right)_{i+1 / 2, j}=\left(q_{l-1}^{\text {new }}\right)_{i+1 / 2, j}-\left(q_{l-1}^{\text {old }}\right)_{i+1 / 2, j}, \\
& \left(\Delta q_{l-1}\right)_{i, j-1 / 2}=\left(q_{l-1}^{\text {new }}\right)_{i, j-1 / 2}-\left(q_{l-1}^{\text {old }}\right)_{i, j-1 / 2}, \\
& \left(\Delta q_{l-1}\right)_{i, j+1 / 2}=\left(q_{l-1}^{\text {new }}\right)_{i, j+1 / 2}-\left(q_{l-1}^{\text {old }}\right)_{i, j+1 / 2},
\end{aligned}
$$

where the cell face states are computed in an upwind manner. We notice that instead of the present procedure of computing coarse grid cell face states in an upwind manner and from that, in a central manner, fine grid cell centre corrections, might as well have been the reverse: computing the coarse grid cell face states in a central manner and from that the fine grid cell centre corrections in an upwind manner. However, a drawback of the latter approach is that it requires additional geometrical data for the upwind computation of the fine grid cell centre corrections.

Given a left and right cell face state ( $q_{\text {left }}$ and $\left.q_{\text {right }}\right)$, for a general 1D upwind scheme, a cell face state $q_{\text {face }}$ may be computed from

$$
f\left(q_{\text {face }}\right)=F\left(q_{\text {left }}, q_{\text {right }}\right),
$$

where $f(q)$ and $F\left(q_{\text {left }}, q_{\text {right }}\right)$ denote the exact and numerical Euler flux function, respectively. A drawback of the Euler equations is that obtaining a primitive state vector like e.g. $q=$ $(\rho, u, \nu, p)^{\mathrm{T}}$ from

$$
f(q)=\left(\rho u, \rho u^{2}+p, \rho u \nu, \rho u(e+p / \rho)\right)^{\mathrm{T}}
$$

requires the solution of a quadratic algebraic equation. Fortunately, with the P-variant of Osher's scheme [3], for most Riemann-problem cases arising in aeronautics $F\left(q_{\text {left }}, q_{\text {right }}\right)=f\left(q_{*}\right), q_{*}$ being a well-defined, single state vector on the Osher path, connecting $q_{\text {left }}$ and $q_{\text {right }}$ in state space. Hence, with the P-variant, in most cases-without evaluating $F\left(q_{\text {left }}, q_{\text {right }}\right)$-we can directly identify $q_{\text {face }}$ as $q_{\text {face }}=q_{*}$. For the O-variant of Osher's scheme [3], in almost all Riemann-problem cases arising in aeronautics $F\left(q_{\text {left }}, q_{\text {right }}\right)$ is found to be the sum of three different fluxes $f(q)$. In these cases, because of $f(q)$ 's nonlinearity, the previous simple procedure is not possible. In the (rare) cases where the P-variant also leads to a sum of fluxes, we solve the quadratic equation and in case of a positive discriminant and one zero being physically irrelevant (negative $\rho$ and/or $p$ ), we take the zero which is physically relevant (positive $\rho$ and $p$ ). In all other cases, we simply take $q_{\mathrm{face}}=\frac{1}{2}\left(q_{\text {left }}+q_{\text {right }}\right)$. Because of the consistency of Osher's scheme at boundaries, there the present upwind prolongation can also be applied in a consistent way. Notice that the upwind prolongation may lead to cell face states which are local extrema in state space. In conclusion, we emphasize that by replacing the piecewise constant prolongation operator by the present upwind prolongation operator, the complete numerical method has become more consistent. Both the discrete Euler operator and the correction prolongation operator are upwind now, both being based on the same upwind scheme: the P-variant of Osher's scheme. 


\subsubsection{Restriction}

A provable consequence of the upwind prolongation is that-unfortunately-no restriction operator $I_{l}^{l-1}$ can be made for which the coarse grid finite volume discretization is a formal Galerkin approximation of the fine grid finite volume discretization [3]. The possibly most effective restriction operator that can be really made is the exact adjoint of the nonlinear prolongation operator. Unfortunately - as opposed to the upwind prolongation - the exactly adjoint restriction operator will certainly lead to a significant increase of the computational overhead. More suitable seems to be a linear approximation of the exact (nonlinear) adjoint. For this we write the latest obtained coarse grid cell face states as linear combinations of the corresponding left and right states:

$$
\begin{aligned}
& \left(q_{\mathrm{face}}\right)_{k}=a_{k}\left(q_{\text {left }}\right)_{k}+\left(1-a_{k}\right)\left(q_{\text {right }}\right)_{k}, \quad k=1,2,3,4, \\
& a_{k}=\frac{\left(q_{\text {face }}\right)_{k} /\left(q_{\text {right }}\right)_{k}-1+\frac{1}{2} \varepsilon}{\left(q_{\text {left }}\right)_{k} /\left(q_{\text {right }}\right)_{k}-1+\varepsilon}, \quad \varepsilon \ll 1,
\end{aligned}
$$

where $q$ is the conservative state vector, $q=(\rho, \rho u, \rho \nu, \rho e)^{\mathrm{T}}$, and $\varepsilon$ a small parameter which guarantees that $\left(q_{\mathrm{face}}\right)_{k}$ is a central average in case $\left(q_{\text {left }}\right)_{k}=\left(q_{\text {right }}\right)_{k}=\left(q_{\text {face }}\right)_{k}$. With next the central computation of the fine grid cell centre states, we then have

$$
\begin{aligned}
& \left(q_{l}\right)_{2 i-1,2 j-1, k}=\left(\tilde{I}_{l-1}^{l} q_{l-1}\right)_{2 i-1,2 j-1, k} \\
& =\frac{1}{2}\left(a_{l-1}\right)_{i-1 / 2, j, k}\left(q_{l-1}\right)_{i-1, j, k} \\
& +\frac{1}{2}\left[2-\left(a_{l-1}\right)_{i-1 / 2, j, k}-\left(a_{l-1}\right)_{i, j-1 / 2, k}\right]\left(q_{l-1}\right)_{i, j, k} \\
& +\frac{1}{2}\left(a_{l-1}\right)_{i, j-1 / 2, k}\left(q_{l-1}\right)_{i, j-1, k}, \quad k=1,2,3,4, \\
& \left(q_{l}\right)_{2 i-1,2 j, k}=\left(\tilde{I}_{l-1}^{l} q_{l-1}\right)_{2 i-1,2 j, k} \\
& =\frac{1}{2}\left(a_{l-1}\right)_{i-1 / 2, j, k}\left(q_{l-1}\right)_{i-1, j, k} \\
& +\frac{1}{2}\left[1-\left(a_{l-1}\right)_{i-1 / 2, j, k}+\left(a_{l-1}\right)_{i, j+1 / 2, k}\right]\left(q_{l-1}\right)_{i, j, k} \\
& +\frac{1}{2}\left[1-\left(a_{l-1}\right)_{i, j+1 / 2, k}\right]\left(q_{l-1}\right)_{i, j+1, k}, \quad k=1,2,3,4 \text {, } \\
& \left(q_{l}\right)_{2 i, 2 j-1, k}=\left(\tilde{I}_{l-1}^{l} q_{l-1}\right)_{2 i, 2 j-1, k} \\
& =\frac{1}{2}\left[1-\left(a_{l-1}\right)_{i+1 / 2, j, k}\right]\left(q_{l-1}\right)_{i+1, j, k} \\
& +\frac{1}{2}\left[1+\left(a_{l-1}\right)_{i+1 / 2, j, k}-\left(a_{l-1}\right)_{i, j-1 / 2, k}\right]\left(q_{l-1}\right)_{i, j, k} \\
& +\frac{1}{2}\left(a_{l-1}\right)_{i, j-1 / 2, k}\left(q_{l-1}\right)_{i, j-1, k}, \quad k=1,2,3,4 \text {, } \\
& \left(q_{l}\right)_{2 i, 2 j, k}=\left(\tilde{I}_{l-1}^{l} q_{l-1}\right)_{2 i, 2 j, k} \\
& =\frac{1}{2}\left[1-\left(a_{l-1}\right)_{i+1 / 2, j, k}\right]\left(q_{l-1}\right)_{i+1, j, k} \\
& +\frac{1}{2}\left[\left(a_{l-1}\right)_{i+1 / 2, j, k}+\left(a_{l-1}\right)_{i, j+1 / 2, k}\right]\left(q_{l-1}\right)_{i, j, k} \\
& +\frac{1}{2}\left[1-\left(a_{l-1}\right)_{i, j+1 / 2, k}\right]\left(q_{l-1}\right)_{i, j+1, k}, \quad k=1,2,3,4 \text {. }
\end{aligned}
$$


With for $q$ the conservative state vector $q=(\rho, \rho u, \rho \nu, \rho e)^{\mathrm{T}}$, the linear relations $(2.14 \mathrm{a})-(2.14 \mathrm{~d})$ approximately, but clearly display how the upwind prolongation distributes mass, momentum and energy from a coarse grid to the overlying finer grid. For the approximately adjoint restriction operator (i.e. the approximation of the exact nonlinear adjoint), we can then write

$$
\begin{aligned}
\left(r_{l-1}\right)_{l, j, k}= & \left(I_{l}^{l-1} r_{l}\right)_{i, j, k} \\
= & \frac{1}{2}\left[1-\left(a_{l-1}\right)_{i-1 / 2, j, k}\right]\left[\left(r_{l}\right)_{2 i-2,2 j-1, k}+\left(r_{l}\right)_{2 i-2,2 j, k}\right] \\
& +\frac{1}{2}\left[1-\left(a_{l-1}\right)_{i, j-1 / 2, k}\right]\left[\left(r_{l}\right)_{2 i-1,2 j-2, k}+\left(r_{l}\right)_{2 i, 2 j-2, k}\right] \\
& +\frac{1}{2}\left(a_{l-1}\right)_{i+1 / 2, j, k}\left[\left(r_{l}\right)_{2 i+1,2, j-1, k}+\left(r_{l}\right)_{2 i+1,2 j, k}\right] \\
& +\frac{1}{2}\left(a_{l-1}\right)_{i, j+1 / 2, k}\left[\left(r_{l}\right)_{2 i-1,2 j+1, k}+\left(r_{l}\right)_{2 i, 2 j+1, k}\right] \\
& +\frac{1}{2}\left[2-\left(a_{l-1}\right)_{i-1 / 2, j, k}-\left(a_{l-1}\right)_{i, j-1 / 2, k}\right]\left(r_{l}\right)_{2 i-1,2 j-1, k} \\
& +\frac{1}{2}\left[1-\left(a_{l-1}\right)_{i-1 / 2, j, k}+\left(a_{l-1}\right)_{i, j+1 / 2, k}\right]\left(r_{l}\right)_{2 i-1,2 j, k} \\
& +\frac{1}{2}\left[1+\left(a_{l-1}\right)_{i+1 / 2, j, k}-\left(a_{l-1}\right)_{i, j-1 / 2, k}\right]\left(r_{l}\right)_{2 i, 2 j-1, k} \\
& +\frac{1}{2}\left[\left(a_{l-1}\right)_{i+1 / 2, j, k}+\left(a_{l-1}\right)_{i, j+1 / 2, k}\right]\left(r_{l}\right)_{2 i, 2 j, k}, \quad k=1,2,3,4 .
\end{aligned}
$$

Of course, the weak spot in (2.13)-(2.15) is the linear approximation of the nonlinear prolongation. In case $\left(q_{\mathrm{face}}\right)_{k}$ is a local extremum, i.e. does no lie in between $\left(q_{\text {left }}\right)_{k}$ and $\left(q_{\text {right }}\right)_{k}$, we have a negative coefficient in (2.13a) (either $a_{k}$ or $1-a_{k}$ ) and hence also in (2.15). We do not accept this situation. If occurring, locally and for that $k$ th component only, we neglect how the upwind prolongation really was and simply consider $\left(q_{\text {face }}\right)_{k}=1 / 2\left(\left(q_{\text {left }}\right)_{k}+\left(q_{\text {right }}\right)_{k}\right)$.

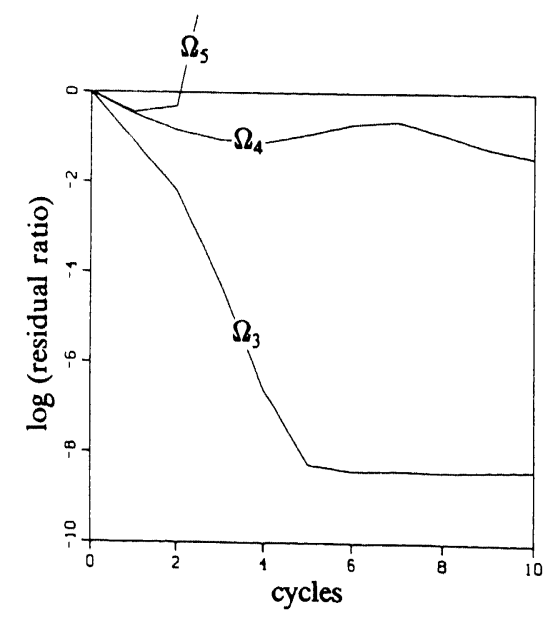

Fig. 7. Multigrid convergence behaviour, blunt forebody with canopy at $M_{\infty}=8.15, \alpha=0^{\circ}$, without nested iteration and without any novel multigrid constituent. 


\section{Numerical results}

We proceed by evaluating the novel techniques proposed. Instead of the multigrid results presented in Fig. 4, the multigrid results shown in Fig. 7 will be used as the starting point for improvement. The multigrid behaviour presented in Fig. 7 is obtained without nested iteration: $q_{l}^{0}, l=1,2, \ldots, L$, are taken uniformly constant and equal to the hypersonic upstream boundary conditions. In this way we obtain a poorer initial approximation, but we have a more discriminating test problem and we are ensured of an unambiguous evaluation, since $q_{l}^{0}, l=$ $1,2, \ldots, L$, will be the same for the different strategies to be considered.

\subsection{With defect multiplication}

With the defect multiplication according to (2.8), damping only, we obtain the convergence results given in Fig. 8(a). With the defect multiplication according to (2.9), both damping and amplification, we obtain the convergence results given in Fig. 8(b). For the matrix norms, in both (2.8) and (2.9), we applied the Frobenius-like norm

$$
\left\|\left(N_{l}^{\prime}\right)_{2 i, 2 j}\right\|=2^{l}\left[\sum_{k_{1}=1, k_{2}=1}^{4,4}\left(N_{l}^{\prime}\right)_{k_{1}, k_{2}}^{2}\right]_{2 i, 2 j}^{1 / 2},
$$

the factor $2^{l}$ simply accounting for the fact that in our case $N_{l}\left(q_{l}\right)$ is a line integral form. Corresponding to the results in Figs. 8(a) and 8(b) in Figs. 9(a) and 9(b) we show distributions of the multiplication operator $S_{L-1}, L=5$ (the multiplication factor distribution on $\Omega_{4}$ ), as applied in the last (i.e. the 10th) multigrid cycle. In both cases the local damping is confined to only the close neighbourhood of the blunt body. Locally, in Fig. 9(b), the damping appears to be a little bit stronger than that in Fig. 9(a). However, globally this is more or less compensated by the local amplifications. Notice that the maximal amplification factor that was found to be applied in Fig. 9(b) is only $\mathrm{O}(1)$. The minimal damping factors in Figs. 9(a) and 9(b) are much larger than those found by De Zeeuw [1] for his specific test case. A second difference with the results
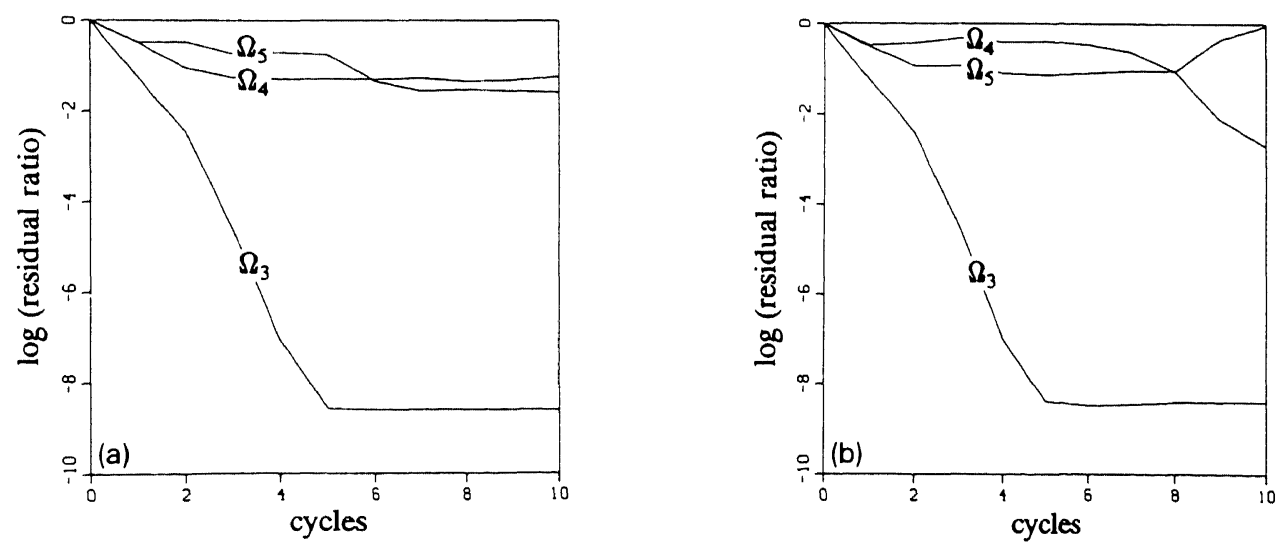

Fig. 8. Multigrid convergence behaviour, blunt forebody with canopy at $M_{\infty}=8.15, \alpha=0^{\circ}$, with defect multiplication according to (a) (2.8), (b) (2.9). 

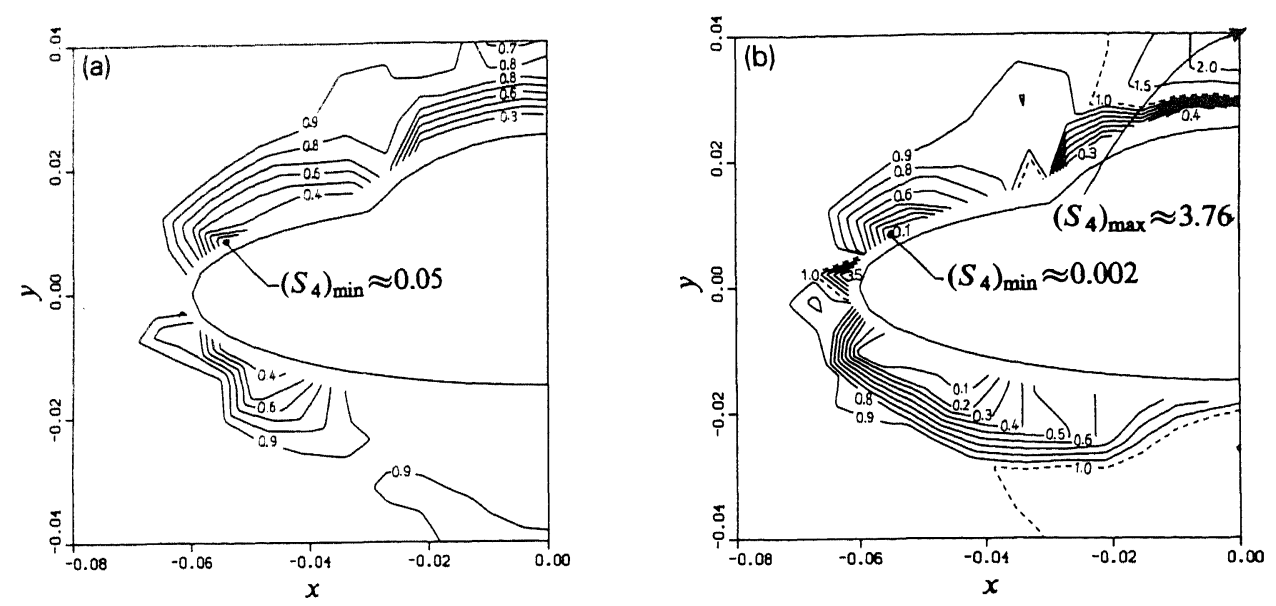

Fig. 9. Distribution multiplication factors applied on $\Omega_{4}$ in the 10th multigrid cycle, blunt forebody with canopy at $M_{\infty}=8.15, \alpha=0^{\circ}$, according to (a) (2.8), (b) (2.9).

of De Zeeuw is the good improvement in [1] of the multigrid method's performance and the modest present improvements. Both differences suggest that (at least) for the present test case, in order to significantly improve the results presented in Fig. 7, defect multiplication is not needed as much as improved grid transfers.

\subsection{With direction-dependent grid transfer operators}

The multigrid behaviour obtained after having replaced both the existing correction prolongation operator and the existing defect restriction operator by the direction-dependent operators, is given in Fig. 10(a). (We notice that defect damping is not applied.) With better grid transfers only, the improvement with respect to Fig. 7 is significant indeed.

Replacing in the existing multigrid algorithm only the standard correction prolongation (by the upwind prolongation), we obtain the multigrid performance given in Fig. 10(b). These results
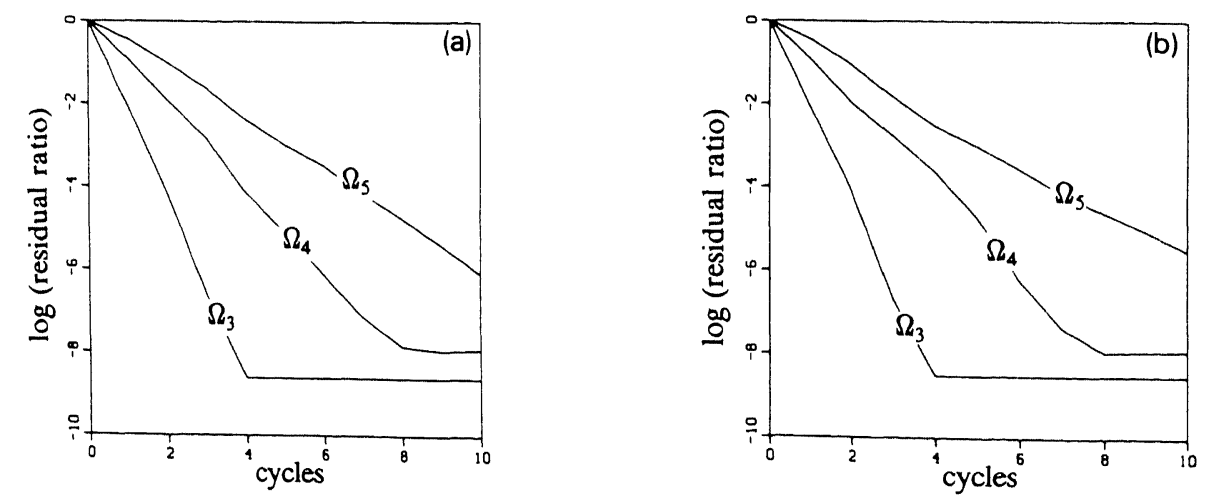

Fig. 10. Multigrid convergence behaviour, blunt forebody with canopy at $M_{\infty}=8.15, \alpha=0^{\circ}$, (a) with upwind correction prolongation and upwind defect restriction, (b) with upwind correction prolongation only. 
are only a little bit less good than those in Fig. 10(a) and hence make us conclude that the previous, rather cumbersome efforts in also upwinding the defect restriction, do not pay off enough. Therefore, in the following we refrain from applying the upwind restriction.

\subsection{With both defect damping and upwind correction prolongation}

Though the two-grid convergence analysis in Section 2.1 assumes that the prolongation operator is linear, see (2.4), no reason exists why local defect multiplication would have a detrimental effect in combination with the nonlinear upwind prolongation. Therefore, in the present section, we show the multigrid performance for the combination of both defect multiplication and upwind correction prolongation. Because the results in Fig. 8(b) already showed not to be better than those in Fig. 8(a) and because of the potential danger for divergence which is inherent to the allowance for local defect amplification, in the following-for the defect multiplication - we will apply (2.8) only.

Combining both novel multigrid constituents as considered separately in the two previous sections, we obtain the multigrid results presented in Fig. 11. Comparison with Fig. 10(b) learns that the combination of both techniques yields a slightly better multigrid performance. We proceed with further investigating the combination. In Fig. 12(a) we show the distribution of the operator $S_{L-1}, L=5$, as applied in the last (i.e. again the 10th) multigrid cycle; a cycle in which the solution has already converged. First we notice that the damping has not vanished indeed. Further we notice that though the solution must be symmetrical around the front ellipse, the damping factor distribution is not. Cause of this is the fact that in the coarse grid problems, for the initial iterate, we take the latest iterate computed. (See e.g. the description of the nonlinear multigrid iteration in Section 1.2 and more explicitly: equation (2.2).) By using the latest obtained iterate, the influence of the very first iterates is still felt, iterates which-due to their poor level of convergence-are not yet symmetrical around the front ellipse. An experimental proof of this explanation is given in Fig. 12(b) in which we show the converged damping factor distribution for a strategy with solution restriction. Here, the converged damping factor distribution around the front ellipse is clearly symmetrical indeed. Notice that in both Fig. 12(a) and Fig.

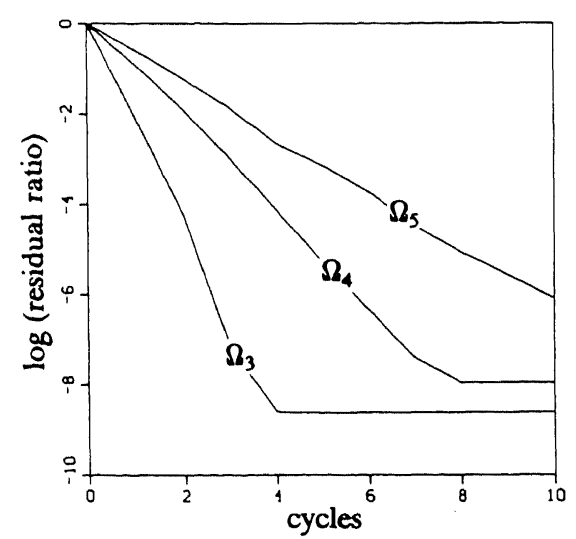

Fig. 11. Multigrid convergence behaviour, blunt forebody with canopy at $M_{\infty}=8.15, \alpha=0^{\circ}$, with both defect damping and upwind correction prolongation. 

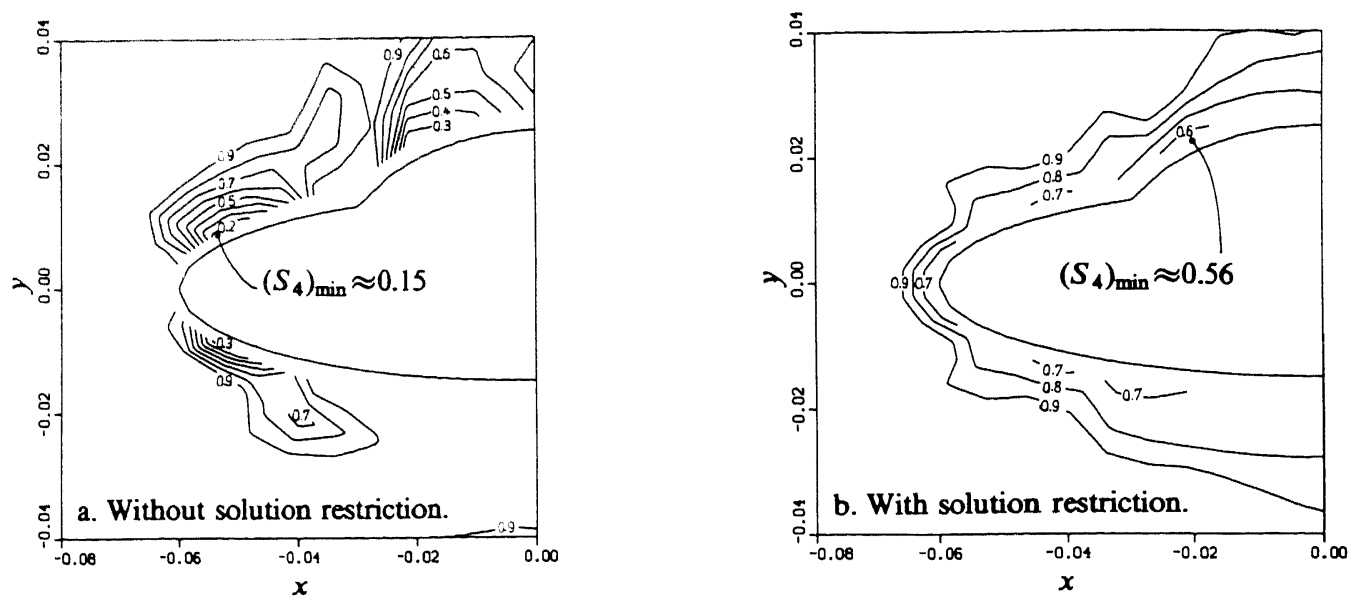

Fig. 12. Converged damping factor distributions on $\Omega_{4}$, blunt forebody with canopy at $M_{\infty}=8.15, \alpha=0^{\circ}$, with both defect damping and upwind correction prolongation.

12(b) the applied damping is modest. In Fig. 12(b) - the case with solution restriction -it is even weaker than in Fig. 12(a). However, taking the restriction of the solution on the coarser grids usually leads to a slower convergence than taking the latest available coarse grid iterates.

\subsection{With both defect damping, upwind correction prolongation and nested iteration}

Comparison of the existing method's results presented in Fig. 4 and Fig. 7-results obtained with and without nested iteration, respectively-clearly shows the natural beneficial influence of nested iteration. For the favourite multigrid strategy, the strategy with defect damping and upwind correction prolongation, the benefit of nested iteration (consistently, with upwind
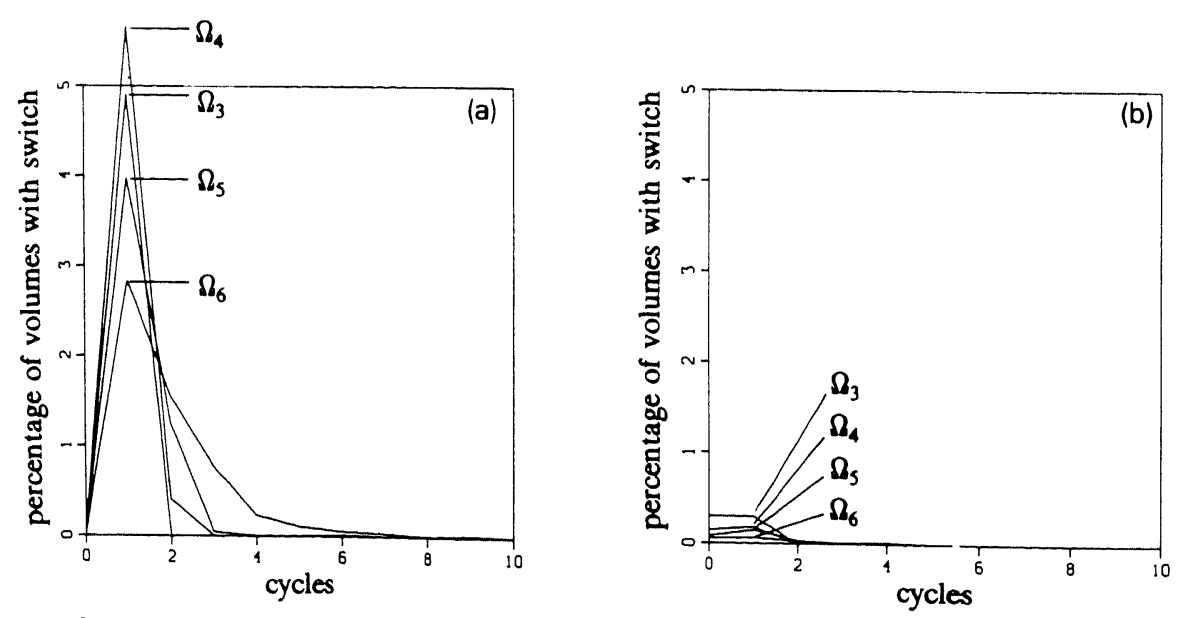

Fig. 13. Amount of volumes with switch from relaxation to evolution, percentage of volumes visited during one multigrid cycle, blunt forebody with canopy at $M_{\infty}=8.15, \alpha=0^{\circ}$, (a) without nested iteration, (b) with nested iteration. 


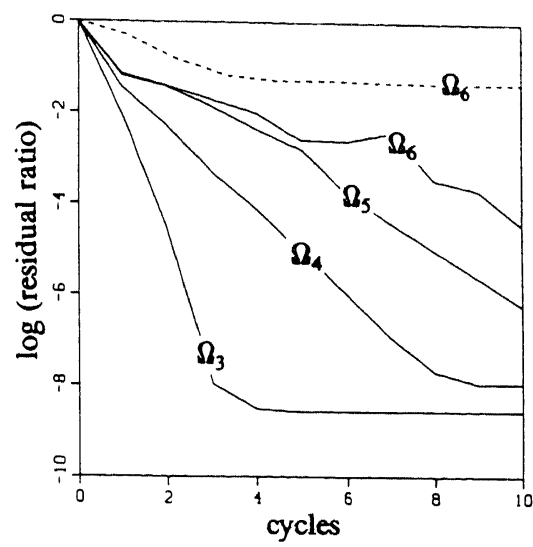

Fig. 14. Multigrid convergence behaviour, blunt forebody with canopy at $M_{\infty}=8.15, \alpha=0^{\circ}$, with both defect damping, upwind prolongation and nested iteration. (Dashed line: single-grid, solid lines: multigrid.)

solution prolongation) is observed by counting the number of finite volumes in which-locallythe switch is made from the relaxation technique to the evolution technique (Fig. 13). In both Figs. 13(a) and 13(b) (without and with nested iteration, respectively), the quantity along the vertical axis is a scaled number of switches made during the $n$th multigrid cycle $(n=1,2, \ldots, 10)$, the scaling factor being the total number of volumes visited during one nonlinear multigrid cycle; a V-cycle with $n_{\text {pre }}=n_{\text {post }}=1$ and with symmetric relaxation sweeps. (Notice that the scaling factor increases when going from $\Omega_{3}$ to $\Omega_{6}$.) The nonzero percentage at $n=0$ in Fig. 13(b) indicates the total amount of switches made during the nested iteration. For all four grids considered, the expected positive influence of the nested iteration appears to be significant. For both strategies the percentage of switches decreases with decreasing mesh size, which indicates that the difficulties are only of a local nature. In Fig. 14 we give the convergence behaviour corresponding with the latest favourite strategy, the strategy with defect damping, upwind
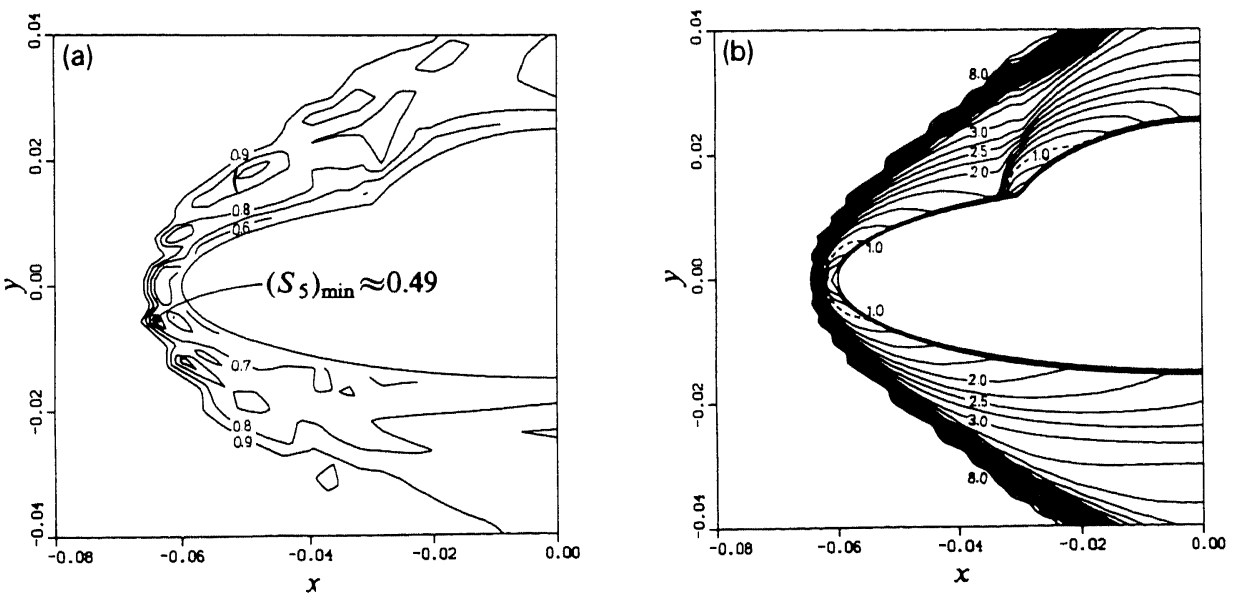

Fig. 15. Converged results, blunt forebody with canopy at $M_{\infty}=8.15, \alpha=0^{\circ}$, with both defect damping, upwind prolongation and nested iteration. (a) Damping factor distribution on $\Omega_{5}$. (b) Mach number distribution on $\Omega_{6}$. 

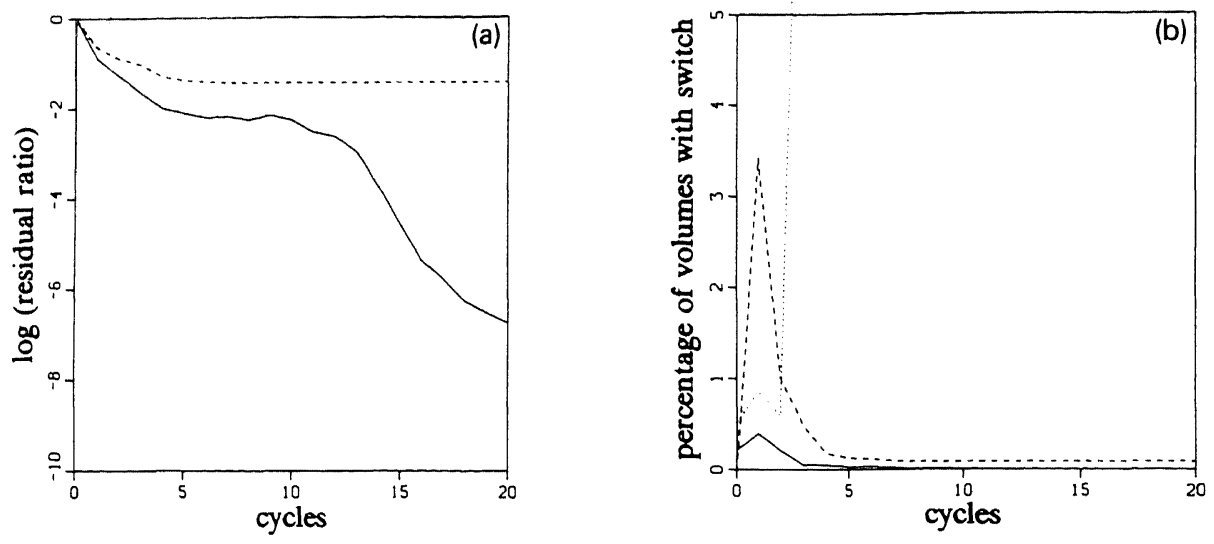

Fig. 16. Multigrid behaviour, blunt forebody with canopy at $M_{\infty}=8.15, \alpha=30^{\circ}$, with both defect damping, upwind prolongation and nested iteration, $\Omega_{6}$ only. (a) Convergence behaviour. (b) Amount of volumes with switch from relaxation to evolution, percentage of volumes visited during one multi-/single-grid cycle. (Dashed line: single-grid, dotted line: already existing multigrid, solid line: novel multigrid.)

prolongation and nested iteration. In this figure, for $\Omega_{6}$, a comparison is also made with the corresponding single-grid convergence behaviour. In Fig. 15 we show a converged damping factor and Mach number distribution. Notice that the smallest damping factors are mainly concentrated along the bow shock, in particular there where the jumps across the shock are largest. Finally, we show results for the more interesting reentry case $M_{\infty}=8.15, \alpha=30^{\circ}$. Also for this test case, the convergence results (Fig. 16) show the beneficial influence of the changes in the existing multigrid method. Given the very low convergence rate of the single-grid computation (Fig. 16(a)) and given the absolute failure of the already existing multigrid method (both
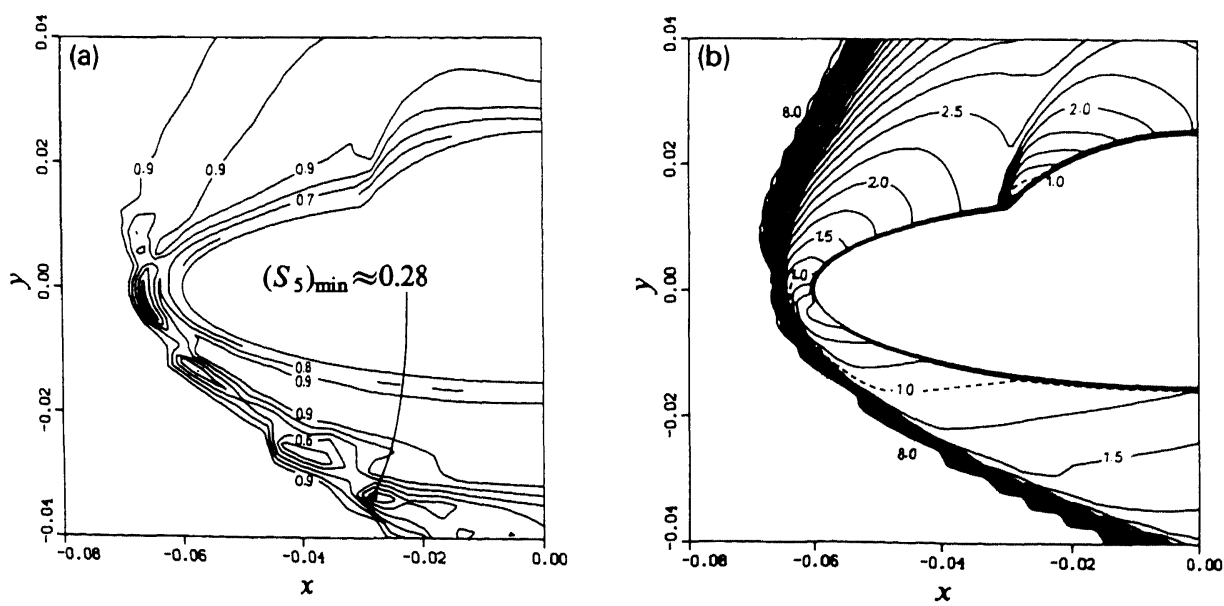

Fig. 17. Converged results, blunt forebody with canopy at $M_{\infty}=8.15, \alpha=30^{\circ}$, with both defect damping, upwind prolongation and nested iteration. (a) Damping factor distribution on $\Omega_{5}$. (b) Mach number distribution on $\Omega_{6}$. 
Figs. 16(a) and 16(b)), the novel multigrid constituents do not just appear to be a nice luxury, but a real necessity. Analogous to Fig. 15, in Fig. 17 we show a converged damping factor and Mach number distribution. Notice that-like in Fig. 15-the smallest damping factors are located at the most pronounced part of the bow shock. Concerning the efficiency of the improved multigrid method, one may find the paradoxical result that one multigrid cycle with both defect damping and upwind correction prolongation is still cheaper than one multigrid cycle without both. The cause of this simply is that the improved multigrid method may result in a significantly smaller number of switches from the local relaxation to a local evolution during the smoothing phases and hence in a lower computational cost. Concerning the efficiency of the upwind computation of cell face states (as applied in the upwind prolongation), for the test cases considered it appears that with the P-variant, at almost all cell faces it holds that $F\left(q_{\text {left }}, q_{\text {right }}\right)=f\left(q_{*}\right)$. (For both $\Omega_{6}$-multigrid cases considered in this section, solving a quadratic equation for $q_{\text {face }}$ appears to be necessary at about $1 \%$ of all cell faces only.)

To finish, we summarize the improved multigrid algorithm, the novel elements being indicated in bold.

\section{Nested iteration}

- Choose $q_{1}$.

- Improve $q_{1}$ by a single nonlinear multigrid cycle.

- Transfer the improved approximation $q_{1}$ to $\Omega_{2}$, by applying the upwind prolongation operator.

- Improve $q_{2}$ by a single nonlinear multigrid cycle.

- Continue the previous process until an initial estimate for $q_{L}$ has been obtained by upwind prolongation of $q_{L-1}$.

\section{Nonlinear multigrid iteration}

- Apply $n_{\text {pre }}$ pre-relaxation sweeps to $N_{l}\left(q_{l}\right)=r_{l}$.

- Compute the defect $d_{l}=N_{l}\left(q_{l}\right)-r_{l}$ and restrict it: $d_{l-1}=I_{l}^{l-1} d_{l}$.

- Compute the local damping factors $\left(S_{l-1}\right)_{i, j}$ and damp the restricted defect: $d_{l-1}:=S_{l-1} d_{l-1}$.

- Compute the right-hand side $r_{l-1}=N_{l-1}\left(q_{l-1}\right)-d_{l-1}$.

- Approximate the solution of $N_{l-1}\left(q_{l-1}\right)=r_{l-1}$ by the application of $n_{\sigma}$ nonlinear multigrid cycles.

- Correct the current solution, by applying the upwind prolongation operator.

- Apply $n_{\text {post }}$ post-relaxation sweeps to $N_{l}\left(q_{l}\right)=r_{l}$.

\section{Conclusions}

For the hypersonic test cases considered in this paper, a satisfactory remedy against divergence of nonlinear multigrid appears to be the combination of a (local) damping of the restricted defect and a (global) upwind prolongation of the correction. Besides a positive influence on the robustness of the algorithm, the combination of upwind prolongation and defect damping also has a positive influence on the computational efficiency. Application-in addition-of an (approximately adjoint) upwind restriction operator does not really pay off. For the test cases considered, the best improvement is obtained by the application of the upwind prolongation 
operator. We think that the good beneficial effects that we have seen from the upwind prolongation operator should not be attributed to the upwind operator itself, but rather to the greater upwind consistency achieved throughout the complete numerical method. For sake of clearness, we remark that the separate merits as observed here for the novel multigrid constituents, may well be different for other test cases. Finally, we remark that the new techniques are such that the improved algorithm still does not require any tuning of parameters.

\section{References}

[1] P.M. de Zeeuw, Nonlinear multigrid applied to a 1D stationary semiconductor model, CWI-Rept. NM-R8905, Centre for Mathematics and Computer Science, Amsterdam (1989); also SIAM J. Sci. Statist. Comput. (to appear).

[2] S.K. Godunov, Finite difference method for numerical computation of discontinuous solutions of the equations of fluid dynamics, Cornell Aeronautical Lab. Translation from the Russian, Math. Sbornik 47 (1959) 271-306.

[3] P.W. Hemker and S.P. Spekreijse, Multiple grid and Osher's scheme for the efficient solution of the steady Euler equations, Appl. Numer. Math. 2 (1986) 475-493.

[4] B. Koren, Robustness improvement of point Gauss-Seidel relaxation for steady, hypersonic flow computations, CWI Note NM-N8805, Centre for Mathematics and Computer Science, Amsterdam (1988).

[5] S. Osher and F. Solomon, Upwind difference schemes for hyperbolic systems of conservation laws, Math. Comp. 38 (1982) 339-374.

[6] A.A. Reusken, Convergence analysis of nonlinear multigrid methods, doctoral thesis, State University of Utrecht, Utrecht, Netherlands (1989). 